\title{
Proceedings of the 133rd Semon Club, Otolaryngology Department, Guy's and St Thomas' National Health Service Foundation Trust, London, 11 May 2007
}

\author{
Chairman: Miss E B Chevretton, Consultant ENT Surgeon, Guy's and St Thomas' National Health Service \\ (NHS) Foundation Trust, London \\ Pathologists: Professor L Michaels, University College London, and Dr A Sandison, University College London \\ Radiologist: Dr C Vanderveld, Specialist Registrar, Radiology, Guy's and St Thomas' NHS Foundation Trust, \\ London
}

Secretary: Mr E Ofo, Specialist Registrar, Otolaryngology, Guy's and St Thomas' NHS Foundation Trust, London

\section{Otology and skull base session \\ Chairman: Mr A Fitzgerald-O'Connor}

\section{Discharging ear and neck swelling in a human immunodeficiency virus positive patient}

A A Hassaan, M Chevali, J P Harcourt

From the Charing Cross Hospital, London, UK

\section{Case report}

A 31-year-old, human immunodeficiency virus positive man presented with a purulent discharge from his right ear associated with increasing ear, mastoid and neck pain. Two weeks earlier, he had received both oral and aural antibiotics from his general practitioner, with no improvement.

Examination revealed a warm, tender, cystic swelling medial to the right sternocleidomastoid muscle. A computed tomography scan reported opacification of the right middle ear and mastoid air cells, cortical destruction of the mastoid process, and an abscess medial to the sternocleidomastoid muscle.

These findings were consistent with a Bezold's abscess, in which infection of the mastoid air cells results in erosion of the mastoid tip, with pus tracking along the posterior belly of the digastric muscle to form an abscess. This pus can also track along the carotid sheath to the hypopharynx and mediastinum.

Treatment included an extended post-auricular incision, with drilling of the mastoid tip, evacuation of the abscess and placement of a Yates drain, as well as administration of antibiotics.

Re-exploration two weeks later showed no otitis media, mastoiditis or re-collection of pus. A grommet was placed in the ear and the patient was discharged without the need for mastoidectomy.

\section{Discussion}

Mr Fitzgerald-O'Connor commented that Bezold's abscess may be under-reported rather than rare. Issues arising from this case included possible under-diagnosis of Bezold's abscess due to unfamiliarity by junior doctors, and a possible increased incidence in immuno-compromised patients. Mr Fitzgerald-O'Connor also noted that the extent of mastoidectomy does not differ when treating Bezold's versus Citelli's abscesses.

\section{An unusual form of 'metastatic' aural disease}

A D Morley, M A Shaikh, P M Robinson

From East Kent Hospitals, Canterbury, UK

Case report

A fit, elderly man presented with recurrent otorrhoea, otalgia and worsening moderate mixed deafness.

The patient had significant stenosis of the mid-portion of the external auditory meati, especially on the left. Treatment consisted of microsuction clearance of the ear canal, topical and systemic steroids and antibiotics, and advice on water avoidance.

The right ear settled, but not the left. The patient had normal haematological, biochemical and inflammatory blood tests. He also had controlled small bowel Crohn's disease.

A magnetic resonance imaging scan was performed to investigate worsening unilateral sensorineural hearing loss, and the result was normal. During a left meatoplasty, biopsies were taken from the areas of meatus cicatrisation. Histological analysis showed multiple granulomatous cells with non-caseating central necrosis in the dermis. 'Metastatic' Crohn's disease was initially diagnosed.

Post-operatively, the patient was managed with steroids and infliximab. His presenting symptoms settled completely.

\section{Discussion}

Professor Michaels and Dr Sandison maintained that the term 'metastatic' should be reserved for malignant tumours; rather, this case represented in their opinion a very rare 'extra-intestinal' manifestation of Crohn's disease. They also advised that tuberculosis should be excluded by performing polymerase chain reaction analysis on the histological specimen.

\section{Unilateral middle-ear effusion in association with an unusual post-nasal space mass and cervical} lymphadenopathy

K G Smith, N A Petkar, M E Papesch

From Whipps Cross University Hospital, London, UK

Case report

A 67-year-old Asian woman was referred to our clinic complaining of right-sided deafness and a lump in her neck.

Pure tone audiometry demonstrated a right-sided, $30 \mathrm{~dB}$, conductive hearing loss, and examination revealed 
a right middle-ear effusion. Endoscopic evaluation of the post-nasal space confirmed a large, friable mass, predominantly on the right. Multiple, palpable cervical lymph nodes were also present.

Computed tomography and magnetic resonance imaging illustrated prominence of the nasopharyngeal mucosa and multiple enlarged cervical lymph nodes, the largest at the left submandibular region, measuring $1.5 \mathrm{~cm}$.

Post-nasal space biopsy demonstrated squamous stratified epithelium with no dysplasia, overlying amorphous material which showed a peri-vascular deposition pattern in some areas. This tissue stained positively with Congo red and demonstrated apple-green birefringence under polarised light, pathognomic for amyloid. Fine needle aspiration cytology of the left level I node, closely followed by formal excision biopsy, confirmed amyloid.

Myringotomy and ventilation tube insertion led to normalisation of hearing at three months. The patient was referred to the National Amyloidosis Centre for amyloid typing and to rule out systemic involvement.

\section{Discussion}

Professor Michaels agreed with the histological diagnosis of primary, localised amyloidosis. Mr Fitzgerald-O'Connor commented on the rarity of this condition as regards its involvement of the post-nasal space and cervical lymph nodes, mimicking nasopharyngeal carcinoma.

\section{Splitting the loop}

A D Slovick, J M Fishman, R Palaniappan

From the Royal National Throat, Nose and Ear Hospital, London, UK

\section{Case report}

A 40-year-old man presented with a 10-year history of debilitating headaches, tinnitus and vertigo, precipitated by loud noises and sneezing (Tullio phenomenon). This occurred in the absence of any sensorineural hearing loss. There was no obvious precipitating cause and no history of head trauma. Pure tone audiometry was unremarkable.

Axial and direct coronal computed tomography (CT) scans revealed convincing evidence of dehiscence of the superior semicircular canal on the left, and almost certainly on the right. No other abnormalities were shown. A previous magnetic resonance imaging scan had been reported as normal. The CT findings correlated well with the observed symptoms and signs. The patient was managed conservatively.

\section{Discussion}

The argument in favour of surgery is controversial, and the optimal surgical approach (transmastoid versus middle craniotomy approach), materials and methods utilised (plugging versus resurfacing), and surgical outcomes remain ill-defined. Surgery was recommended only in the presence of debilitating symptoms, supported by classic signs and CT findings. Dr Vanderveld commented that the condition may be over-reported, as current CT scanners may suggest a dehiscence where one does not necessarily exist.

\section{Persistent anterior skull base infection despite resolution of otitis externa}

M Anwar, S A Hannan, W E Grant

From the Charing Cross Hospital, London, UK

Case report

An 83-year-old professor presented with left-sided otorrhoea and otalgia. Serial out-patient management of presumed otitis externa settled the otorrhoea, but otalgia remained and was soon complicated by dysphagia and debility. Oral ciprofloxacin was instituted, presuming a diagnosis of necrotising otitis externa, and investigations followed.

A computed tomography (CT) scan revealed retained secretions in the middle-ear cleft, as expected. It also showed separate soft tissue swelling in the nasopharynx with clival erosion. Subsequent magnetic resonance imaging confirmed extensive, abnormal soft tissue enhancement of the skull base, suggesting low-grade infection or tumour.

Multiple deep post-nasal space biopsies, taken under image-guidance, prompted a sudden release of pus from an abscess cavity. Analysis revealed fibrotic, chronically inflamed, necrotic tissue, but no evidence of malignancy. Special stains for bacteria, fungi, mycobacteria and amyloid were all negative, although microbiological culture of the fluid yielded Pseudomonas aeruginosa.

Further management, decided at a skull base multidisciplinary team meeting, involved prolonged oral ciprofloxacin. Under regular out-patient department review, the patient's usual sprightly demeanour was seen to return. At the time of writing, the most recent follow-up CT revealed normal mastoid air cells and no nasopharyngeal soft tissue mass.

\section{Discussion}

Professor Michaels suggested that this was probably a case of necrotising otitis externa which had migrated to the anterior skull base, and a bone scan may have helped in the diagnosis.

\section{Head and neck session}

Chairman: Mr R Simo

\section{A rare case of recurrence of a right orbital mass}

R McTiernan, A Cunningham, JP Jeannon

From Guy's Hospital, London, UK

\section{Case report}

A 65-year-old man presented to the King's College Hospital ophthalmologists with a mass around the right medial canthus of the eye. Tricholemmal carcinoma was confirmed histologically in December 2004. Positive excision margins were noted, but the patient chose follow up and declined further excision and/or radiotherapy. He re-presented in June 2006 with a recurrent right eye mass and was referred to Guy's Hospital for further management.

A computed tomography scan (June 2006) confirmed recurrence originating from the right medial canthus. Radiological features were consistent with recurrence of tricholemmal carcinoma.

Further biopsies confirmed recurrence of a poorly differentiated tricholemmal carcinoma with an aggressive infiltration pattern along the tract of the previous biopsies, with associated perineural invasion.

The patient underwent a right orbital exenteration, ethmoidectomy, partial rhinectomy, partial maxillectomy, reconstruction using a VRAM rectal myo-cutanous free tissue transfer, orbital implants and adjuvant radiotherapy.

\section{Discussion}

Professor Michaels agreed with the diagnosis of tricholemmal carcinoma. Mr Simo commented that tricholemmal carcinoma is extremely rare, making it difficult to predict tumour behaviour. This case highlights the importance 
of clear excision margins and close follow up. The challenges of managing lesions near the functionally crucial eye region were explored within a tertiary head and neck multidisciplinary team in order to plan resection and reconstruction.

\section{Recurrent 'cyst' of the right parotid gland: a very rare histology}

M Koutra, J Hughes, A Robinson

From the West Middlesex University Hospital, Isleworth, UK

Case report

A 69-year-old woman presented with a six-month history of a swelling in her right parotid area, with no other associated symptoms. The initial clinical assessment, ultrasound examination and fine needle aspiration suggested a benign cyst. The cyst recurred after nine months, and a repeat ultrasound prompted suspicion of a nodular component.

An ultrasound examination of the right parotid gland showed a thin-walled, avascular cyst, which contained an internal septum and a solid nodule attached to the cyst wall.

Following this, the patient underwent a right parotid exploration, and a cystic tumour was excised.

Histological analysis showed a salivary intraductal carcinoma with no invasive component.

Long term clinical and radiological surveillance was advocated after discussion with the multidisciplinary team and the patient.

\section{Discussion}

The number of cases of salivary intraductal carcinoma reported in the literature is too small to judge the potential behaviour of this tumour and the appropriateness of the chosen management option in this case. As the prognosis is uncertain, $\mathrm{Mr}$ Simo recommended post-operative radiotherapy. Other comments from the floor (Mr Vaz) included the opinion that re-exploration would cause significant post-operative morbidity and that a total parotidectomy should therefore be postponed until there was radiological evidence of recurrent disease.

Cutaneous seeding of squamous cell carcinoma of occult primary site following diagnostic fine needle aspiration cytology

P Stimpson, A Joshi, R Simo

From Guy's Hospital, London, UK

\section{Case report}

A 59-year-old woman presented with a three-month history of an enlarging right neck lump. She had a 30 pack year smoking history and alcoholic liver cirrhosis.

At presentation, the patient had a $4 \times 4 \mathrm{~cm}$, level III, right neck lump. Ultrasound-guided fine needle aspiration cytology (FNAC) demonstrated metastatic keratinising squamous cell carcinoma (SCC).

The patient was then referred to our head and neck unit, and was noted to have a $6 \times 6 \mathrm{~cm}$, level III, right neck mass with overlying skin involvement. No primary site was identified on clinical examination. Following appropriate imaging, including computed tomography (CT) and positron emission tomography (PET) scanning, the patient underwent panendoscopy and pan-mucosal biopsies, together with a right modified radical neck dissection.

The CT scan demonstrated an ill-defined, necrotic lymph node mass in the right submandibular region, extending to the skin. A bulky, multiloculated, cystic mass in the right lobe of the thyroid was also noted showing rim enhancement. Scanning with PET-CT did not identify a primary site.

Mucosal biopsies were all negative. The neck dissection specimen demonstrated a poorly differentiated mass of SCC with multiple metastases of a follicular variant of papillary thyroid carcinoma. There was evidence of seeding in the skin overlying the mass from the tract created at the time of FNAC. The final histological diagnosis was a $\mathrm{pT}_{\mathrm{x}} \mathrm{N}_{3} \mathrm{M}_{0} \mathrm{SCC}$ of occult primary site.

The patient subsequently underwent total thyroidectomy and level VI neck dissection, as recommended by the multidisciplinary team. Histological analysis revealed a $\mathrm{pT}_{3} \mathrm{~N}_{1 \mathrm{~b}}$, stage III papillary thyroid carcinoma. The patient completed radioiodine treatment and, at the time of writing, was expected to undergo external beam radiotherapy for the SCC (unknown primary).

\section{Discussion}

Mr Jacob raised the possibility that the cutaneous involvement may have reflected direct tumour spread and would have been unlikely to have resulted from the FNAC. However, a needle track from the lesion to the overlying skin was noted on the original pathology report. Concerns were raised regarding the decision to operate on the neck without further investigating the abnormal thyroid demonstrated on CT scan. Mr Simo explained that this was due to logistical reasons; there were concerns that delayed treatment would have been detrimental over the Christmas period in a patient with such clinically aggressive disease. In conclusion, FNAC is generally safe and effective. However, seeding of disease to the skin does rarely occur. This is the first confirmed case report showing cutaneous seeding of an occult primary to the neck, following FNAC for a metastatic SCC.

\section{Peri-implant squamous cell carcinoma: a second primary in a rehabilitated patient}

B Collard, L Cascarini, J Kwok

From Guy's Hospital, London, UK

\section{Case report}

In 2006, a 67-year-old woman presented with a two-week history of an ulcer around a dental implant. She had previously been treated for two small squamous cell carcinomas on the lateral border of the tongue, in 2001 and 2004. She had smoked approximately 40 cigarettes a day for 30 years but had given up smoking in 1997; she also consumed seven units of alcohol per week.

A biopsy revealed squamous cell carcinoma (SCC). The tumour was clearly associated with the implant and could not be considered a recurrent tumour. Plain film radiographs showed no bony abnormality around the implant.

The patient underwent a segmental mandibular resection and level I-IV selective neck dissection. Reconstruction employed a composite radial forearm free flap. The SCC was completely excised, with no evidence of bony invasion or metastatic spread.

\section{Discussion}

Dental implants are used for dental rehabilitation after oral cancer surgery. Although it is difficult to know whether this patient would have developed a malignancy in the absence of dental implants, this case raises the possibility that, in patients at risk, irritation around implants may act as an initiating factor in the development of oral SCC. However, the panel felt that this would be a very rare 
event, and that the use of implants in such patients should continue.

\section{An unusual case of cervical lymphadenopathy and night sweats}

C Loh, G A J Morrison

From Guy's and St Thomas' Hospital, London, UK

Case report

A 28-year-old, Afro-Caribbean woman presented with a four-week history of bilateral cervical lymphadenopathy, fevers and night sweats. There was no preceding coryzal illness or upper respiratory tract infection.

Examination revealed no abnormality other than cervical lymphadenopathy. Ultrasound-guided core biopsies were inconclusive, and an excision biopsy was performed.

Histological analysis revealed reactive lymphoid hyperplasia with focal collections of histiocytic cells with prominent apoptosis. The cells were positive for CD68 and myeloperoxidase. There was an associated infiltrate of CD8 T-cells. The features were consistent with Kikuchi disease (histiocytic necrotising lymphadenitis).

The lymphadenopathy began to resolve, and at the time of writing the patient was being kept under review.

\section{Discussion}

Kikuchi disease is an uncommon, idiopathic, generally selflimiting cause of lymphadenitis. There are only isolated cases in Europe, but a higher prevalence in Asia. Kikuchi disease can be mistaken for malignant lymphoma clinically, and its differentiation from lymphoma is difficult, even histopathologically. The disease is also sometimes associated with systemic lupus erythematosus.

Professor Michaels reiterated that fact that once a histological diagnosis of Kikuchi disease is made, investigations for other causes of lymphadenopathy are not needed. Recognising the features of Kikuchi disease is important in order to avoid misdiagnosis, as there have been reports of Kikuchi disease being initially misdiagnosed as lymphoma, with patients being overtreated with chemotherapy as a consequence.

\section{Rhinology session}

Chairman: Mr D Roberts

Cementifying, ossifying fibroma of the left nasal cavity: revisiting the fibro-osseous diagnostic dilemma

A Joshi, R Oakley, D Roberts

From Guy's Hospital, London, UK

\section{Case report}

A 44-year-old man presented with left-sided facial pain and puffiness of the left eye. Interestingly, he had no nasal symptoms.

However, on examination, there was a friable, bony mass filling the left nasal cavity.

Computed tomography and magnetic resonance imaging scans showed an expanding, well defined, lobulated mass displacing the septum, along with extension into the left maxillary and ethmoidal air cells, around an area of irregular calcification. The differential diagnosis was considered to include mucocele with fungal changes, chordoma and mucous gland tumour.

However, histological analysis showed spindle cells with numerous cementum-like bodies and no atypia, consistent with a diagnosis of cementifying, ossifying fibroma.
Subsequently, an endoscopic radical clearance of the mass was performed up to the orbital apex and sphenoid sinus.

\section{Discussion}

Ossifying fibromas are benign, painless, slow-growing, monostotic tumours seen more commonly in women between the second and fourth decades of life, and exclusively affecting the cranial bones. Patients commonly present with a painless swelling of the cheek, unilateral proptosis with diplopia, persistent nasal obstruction, rhinorrhoea, epiphora, and recurrent epistaxis and haemoptysis. Differentiation of the ossifying fibroma from fibrous dysplasia is of importance, as cessation of growth is unlikely and aggressive en bloc resection is advisable initially in order to prevent progression and compression of cranial nerves around the skull base. Genetic analysis of a number of patients with intragnathic ossifying fibroma has shown mutation of HRPT2, which, alongside the GNAS1 mutation found in fibrous dysplasia, may allow the development of a clearly defined genetic subclassification of fibro-osseous lesions. Professor Michaels commented that the origin of these tumours is unknown, and that they have a strong tendency to recur.

\section{Two cases of an unusual nasal skin lesion presenting as more common pathologies}

C Pepper, S Baer, P Kirkland

From the Conquest Hospital, East Sussex NHS Trust, St Leonards-on-Sea, UK

Winner of prize for best presentation

\section{Case report one}

A 59-year-old woman presented with a three-month history of a $1 \mathrm{~cm}$ nodule on the left side of the nose, clinically resembling a basal cell carcinoma. The lesion was excised under local anaesthesia, with local flap reconstruction.

Histological analysis revealed a cutaneous B-cell lymphoma of follicle centre cell origin. Bone marrow aspirate was normal.

\section{Case report two}

A 52-year-old woman presented with a six-month history of an itchy, discharging lump on the left side of her nose, clinically resembling a sebaceous cyst. This was excised under local anaesthesia.

Histological analysis revealed a cutaneous B-cell lymphoma of marginal zone origin. Bone marrow aspirate was also normal.

In both cases, contrast computed tomography scanning of the neck, thorax, abdomen and pelvis showed no evidence of metastases, and the patients were managed with periodic follow up and review by the haematology team.

\section{Discussion}

These cases describe two skin lesions presenting clinically as a basal cell carcinoma and a sebaceous cyst. However, careful histological examination revealed both lesions to be primary cutaneous B-cell lymphoma, of follicle centre and marginal zone types, variously. Dr Sandison agreed with these diagnoses. Mr Roberts commented on two similar cases of primary cutaneous B-cell lymphoma on the nose, which he had also managed with excision and close follow up. It is important to bear in mind that unusual cutaneous pathologies can present as common skin conditions on the nose. Therefore, it is important to send all excised lesions for histological evaluation. 


\section{An unusual case of a large, crusty nose}

S Esprit, H Sharif, N Bleach

From the Wexham Park Hospital, Slough, UK

\section{Case report}

Over a six-week period, a 39-year-old man developed diffuse, massive, painless swelling of his nasal tip and alar margins, with crusting, oozing and abscess formation. After three courses of oral antibiotics plus application of two topical antibiotic creams, his symptoms showed no improvement.

Blood tests revealed a negative autoimmune screen, an erythrocyte sedimentation rate of 28 , a C-reactive protein level of 25, moderate eosinophilia, neutrophilia and negative results for blood-borne virus tests.

After discussion with the microbiologists, the patient showed moderate improvement with a week's course of

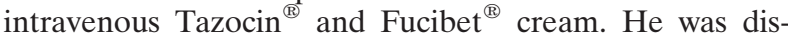
charged home on oral ciprofloxacin.

However, within two weeks the patient's symptoms escalated, and he was readmitted. He underwent two examinations under general anaesthesia within a one-month period. Biopsies were sent for histological and microbiological examination.

Histological examination revealed pseudo-epitheliomatous hyperplasia, eosinophila and microabscess formation.

At the time of writing, the patient's symptoms were controlled with oral Roaccutane ${ }^{\circledR} 40 \mathrm{mg}$ combined with prednisolone $20 \mathrm{mg}$; however, he still had residual nasal swelling and may require dapsone or tumour necrosis factor inhibitors. A diagnosis was not yet established. Rosacea fulminans was the most similar condition histologically, although the patient's clinical picture was atypical for this condition.

\section{Discussion}

This patient was particularly interesting as he presented with unusual symptoms and had unexpected histology. After reviewing the histology slides, Professor Michaels could not arrive at a definitive diagnosis.

\section{A three-month history of nasal obstruction}

A Cunningham, R McTiernan, D Roberts

From Guys Hospital, London, UK

\section{Case report}

A 41-year-old woman had a three-month history of rightsided nasal symptoms.

On examination, she was found to have a large, rightsided, inflammatory nasal mass associated with pus discharging from the maxillary sinus.

Computed tomography scanning of the paranasal sinuses showed a soft tissue mass completely occluding the patient's right nasal cavity, with extension into the ethmoid air cells and the sphenoid sinus.

At endoscopic resection, the macroscopic appearance was that of a fibrotic tumour arising from the root of the middle turbinate.

Histological analysis showed regions of dense cellularity, stromal sclerosis and focal areas of gland formation. Hence, a diagnosis of biphasic synovial cell sarcoma was made. Immunohistochemical analysis revealed that the cells were positive for AE 1/3, EMA and BCL-2 and negative for CD99, results supportive of a synovial sarcoma immunophenotype.

The patient underwent post-resection radiotherapy, and at the time of writing had suffered no recurrence.

\section{Discussion}

Under 10 per cent of all soft tissue sarcomas are of the synovial type, and of these less than 10 per cent occur in the head and neck. Therefore, this case represents a rare finding of a biphasic synovial sarcoma involving the paranasal sinuses, namely the ethmoid. The biphasic subtype displays a specific genotype which has been mapped to the SSX1 gene, involving the translocation $\mathrm{t}(\mathrm{X}: 18)$ (p11:q11). Only one other case of an ethmoidal synovial sarcoma has been reported, and that was of the monophasic subtype. $\mathrm{Mr}$ Roberts commented on the difficulty of managing these patients, and stressed that close radiological follow up with repeat biopsies is essential.

\section{An unusual differential diagnosis of a unilateral nasal mass}

T Chapman, A Joshi, D R Roberts

From Guy's Hospital, London, UK

\section{Case report}

A 40-year-old, white female presented with a six-year history of nasal obstruction and hyposmia, worsening over the past year. She had previously been treated unsuccessfully with topical nasal steroids.

Nasendoscopy demonstrated a large, polypoidal mass of the right nasal cavity, arising from the middle turbinate and occupying the posterior choanae.

Computed tomography (CT) and magnetic resonance imaging (MRI) confirmed the clinical findings and showed apparent infiltration of the sphenoid bone, with features suggestive of a chondroid tumour such as chondrosarcoma. An endoscopic ethmoidectomy and sphenoidectomy was performed, with macroscopic clearance of the tumour achieved.

Histological analysis revealed a proliferation of glands lined by ciliated respiratory epithelium, characteristic of respiratory epithelial adenomatoid hamartoma. The sphenoid bone was unremarkable. Full resolution of the patient's symptoms was noted at follow up.

\section{Discussion}

Histology slides were unavailable for further analysis at the meeting. Respiratory epithelial adenomatoid hamartoma is a rare, benign, sinonasal/nasopharyngeal lesion first described in 1995. The aetiology of this growth arising from the schneiderian epithelium is unclear, but may be secondary to sinonasal inflammation. The majority of cases affect males, with non-specific presentations including nasal obstruction, deviated septum, epistaxis and recurrent rhinosinusitis. Importantly, CT and MRI show no characteristic signs. The treatment is curative through complete local excision, with no reports of recurrent disease. The primary concern is that respiratory epithelial adenomatoid hamartoma may be confused with inverted papilloma or adenocarcinoma, leading to unnecessarily aggressive surgery. The session chair agreed that this condition displayed rare and interesting pathology with significant potential for misdiagnosis.

\section{Paediatric session}

Chairman: Mr G Morrison

\section{A rapidly expanding mandibular mass in a neonate}

J Ahmed, N J Sebire, B E J Hartley

From the Great Ormond Street Hospital for Children, London, UK

\section{Case report}

A one-week-old male neonate displayed rapid growth of a swelling over the right mandible, resulting in an obvious 
facial and intra-oral swelling, over the course of a few weeks.

At four weeks of age, a computed tomography scan revealed expansion of the ramus of the right mandible with a lytic central area. Magnetic resonance imaging confirmed a $4 \times 3 \times 3 \mathrm{~cm}$ mass abutting the right side of the tongue and extending superiorly into the right parapharyngeal space.

The diagnosis could only be established by histological examination, which looked for the classical appearance of two distinct cell populations: neuroblast-like, small, round cells and pigment-laden, epithelioid cells. In the present case, the histological features varied on the initial incision biopsy and the subsequent resection specimen. The latter had been exposed to chemotherapy, which had apparently eradicated the small, round cell population. Chemotherapy had been administered initially following an incorrect provisional diagnosis of primitive neuroectodermal tumour.

Treatment of this condition was surgical, with chemotherapy reserved for metastatic disease. In our case, surgery consisted of right hemimandibulectomy with primary reconstruction using autologous costochondral rib graft. The surgical management would not have differed even if the provisional diagnosis of chemotherapy-resistant primitive neuroectodermal tumour had been confirmed.

\section{Discussion}

Dr Sandison diagnosed melanocytic neuroectodermal tumour of infancy. The presenter was asked why a repeat biopsy had not been performed prior to surgery, and was told that the original histology slides had been sent by the referring institution. Mr Morrison questioned why a free bone graft had been preferred to a vascularised graft. The authors felt that, although controversial, they would advocate the above method of reconstruction in infants with extensive mandibular continuity defects, because of their positive experience thus far.

\section{Cervical lymphadenopathy with persistent pyrexia: an interesting case}

R J Glore, J Bajwa, A Jacob

From Lewisham Hospital, London, UK

\section{Case report}

A five-year-old, Afro-Caribbean boy was admitted with a two-day history of odynophagia, a firm left neck swelling $(4 \times 4 \mathrm{~cm})$ and spiking fever $\left(40^{\circ} \mathrm{C}\right)$, following an upper respiratory tract infection. The swelling rapidly increased in size over 48 hours but remained non-tender. The child later developed dry, peeling skin over his legs.

The full blood count demonstrated a persistently raised white cell count (31.1-38.5) and C-reactive protein (146268). All viral serology was negative, and the child tested negative for tuberculosis.

Cervical ultrasonography revealed lymphadenopathy but no collection. An abdominal ultrasound was normal, whilst a chest X-ray demonstrated a prominent heart with increased lung markings around the hila and cardiac borders. Echocardiography showed tricuspid and mitral regurgitation.

Although the patient's cervical lymphadenopathy settled completely with intravenous antibiotics and steroids, he remained lethargic and pyrexial. A paediatric opinion was sought, and, on the basis of the patient's continuing pyrexia, red eyes and echocardiogram findings, a diagnosis of atypical Kawasaki's disease was made. The patient improved dramatically with the addition of immunoglobulins and aspirin.

\section{Discussion}

Mr Morrison commented that it is important to consider atypical Kawasaki disease in the differential diagnosis of sick children with neck lumps and pyrexia, as this condition is more common that is currently appreciated.

\section{A rapidly enlarging facial mass in a four-year-old boy}

N Mani, B Hartley

From the Great Ormond Street Hospital for Children, London, UK

\section{Case report}

A four-year-old boy presented with a three-week history of a rapidly enlarging swelling beneath the skin of his left cheek.

Examination revealed a $5 \times 5 \mathrm{~cm}$, firm, non-tender mass in the left maxillary region, extending into the left nasal cavity.

Computed tomography and magnetic resonance imaging showed marked bone erosion extending into the alveolar process of the left maxilla and inferiorly into the hard palate. A core biopsy of the mass was highly suggestive of a malignant peripheral nerve sheath tumour.

Chemotherapy was commenced to shrink the tumour, with a view to subsequent surgical resection.

During the first cycle of chemotherapy, tumour growth continued. Further imaging confirmed progression of the tumour, which was now involving the left orbital floor. A subtotal maxillectomy with en bloc excision of the tumour was performed. Clear resection margins were obtained. The patient received post-operative radiotherapy.

\section{Discussion}

Dr Sandison confirmed the diagnosis of a malignant peripheral nerve sheath tumour. A member of the audience suggested that a midfacial degloving approach might have been suitable. Mr Hartley felt that the Weber-Ferguson approach which had been used gave the best chance of oncological clearance and also allowed for the excision of overlying skin, had this been deemed necessary at the time of surgery. On questioning from Mr Morrison, $\mathrm{Mr}$ Hartley confirmed that these tumours behave similarly to those seen in the adult population. Malignant peripheral nerve sheath tumour is a rare and aggressive neoplasm which has a variable response to chemotherapy. Multimodal therapy offers the best chance of cure.

\section{Reducing peri-operative morbidity in a child undergoing adenotonsillectomy for severe obstructive sleep apnoea}

S S Vasani, A Trinidade, H Caulfield

From the Royal Free Hospital, London, UK

\section{Case report}

A three-year-old Nigerian girl with a history of severe obstructive sleep apnoea (OSA) was listed for adenotonsillectomy. As per protocol, the patient was admitted the night before surgery to enable nurse-led continuous pulse and oxygen saturation monitoring. This demonstrated desaturations to 62 per cent, with an average oxygen saturation of 84 per cent. The operation was cancelled and the child sent home with oral dexamethasone $100 \mu \mathrm{g} / \mathrm{kg}$ once daily and co-amoxiclav $125 / 31 \mathrm{mg}$ thrice daily for two weeks.

On return, a repeat sleep study showed dramatic improvement: saturations did not fall below 98 per cent at any point during the study. The operation went ahead without complications.

Although pre-operative improvements of this magnitude, following medical therapy, are unprecedented in 
the literature, this case was not alone in the department's experience. Colleagues retrieved the sleep studies of four other children with OSA who had been treated with the same regimen; these studies showed similar, highly statistically significant improvements in the children's sleepdisordered breathing pre-operatively.

\section{Discussion}

Mr G Morrison questioned whether this treatment was intended as a cure, but Dr Vasani clarified that it was a means of ameliorating the disease in the peri-operative period, allowing adenotonsillectomy to be performed, even in patients with severe OSA, without paediatric intensive care unit cover. A member of the audience also questioned whether there was any need for surgery if patients responded so well pre-operatively to steroids and antibiotics.

\section{An unusual cause of cough}

R Heywood, L Cochrane, B Hartley

From the Great Ormond Street Hospital for Children, London, UK

Case report

A one-year-old boy presented with an episode of pneumonia. Following this, he suffered from intermittent dyspnoea, which responded well to inhaled salbutamol. At the age of three years, he became acutely unwell, with cough, intermittent fever and weight loss.

Chest X-rays demonstrated near-total 'white out' of the left lung, with only a small amount of aeration at the apex, and hyperinflation of the right lung. Computed tomography scanning showed an endobronchial lesion occluding the left main bronchus, with associated left lower lobe consolidation.

Flexible bronchoscopy revealed a lesion obstructing the left main bronchus just distal to the carina. This was excised from the left main bronchus, using rigid bronchoscopy. There was no evidence of residual or recurrent disease after 18 months of follow up. At the time of writing, the patient continued to be monitored closely.

\section{Discussion}

On histological examination, Dr Sandison was of the opinion that the lesion was an inflammatory myofibroblastic tumour. Inflammatory myofibroblastic tumour is a poorly understood lesion with unpredictable biological behaviour. Surgical resection is generally advocated for diagnosis and treatment. The authors emphasised the use of rigid bronchoscopy as a relatively non-invasive yet successful tool in the diagnosis, treatment and follow up of these lesions. 